\title{
Timing Characteristics of Large Area Picosecond Photodetectors
}

\author{
B.W. Adams ${ }^{\text {a }}$, A. Elagin ${ }^{\text {b }}$ H. Frisch ${ }^{\text {b }}$, R. Obaid ${ }^{\mathrm{c}}$, E. Oberla $^{\mathrm{b}}$, A. Vostrikov $^{\mathrm{b}}$, \\ R. Wagner ${ }^{\mathrm{a}}$, J. Wang ${ }^{\mathrm{a}}$, M. Wetstein ${ }^{\mathrm{b}, *}$ \\ ${ }^{a}$ Argonne National Laboratory \\ ${ }^{b}$ Enrico Fermi Institute, University of Chicago \\ ${ }^{c}$ University of Connecticut
}

\begin{abstract}
The LAPPD Collaboration was formed to develop ultrafast large-area imaging photodetectors based on new methods for fabricating microchannel plates (MCPs). In this paper we characterize the time response using a pulsed, subpicosecond laser. We observe single-photoelectron time resolutions of a $20 \mathrm{~cm}$ x $20 \mathrm{~cm}$ MCP consistently below 70 picoseconds, spatial resolutions of roughly 500 microns, and median gains higher than $10^{7}$. The RMS measured at one particular point on an LAPPD detector is $58 \mathrm{psec}$, with $\pm 1 \sigma$ of $47 \mathrm{psec}$. The differential time resolution between the signal reaching the two ends of the delay line anode is measured to be 5.1 psec for large signals, with an asymptotic limit falling below 2 picoseconds as noise-over-signal approaches zero.

Keywords: MCP, TTS, Single photon, TOF, LAPPD, resolution, picosecond, large area, photodetector
\end{abstract}

\section{Introduction}

Microchannel plate photomultiplier tubes (MCP-PMTs) are compact vacuum photodetectors [1, capable of micron-scale spatial resolutions 2, subnanosecond time resolutions [3, 4, 5], and gains exceeding $10^{7}[\underline{6}$. Economi5 cal, large-area MCP photosensors with these characteristics would bring much

\footnotetext{
${ }^{*}$ Corresponding author

Email address: matt.wetstein@gmail.com (M. Wetstein)
}

Preprint submitted to Nucl. Instrum. Methods A

April 24, 2015 
needed timing and imaging capabilities to a wide range of applications in fields such as particle physics, nuclear physics, X-ray science, and medical imaging.

The Large Area Picosecond Photodetector (LAPPD) collaboration was formed to develop techniques for making large format $(20 \mathrm{~cm}$ x $20 \mathrm{~cm}$ ) MCP-PMT detector systems using scalable methods and low-cost materials, addressing technical aspects of the problem from the photocathode and the gain stage to the readout electronics and vacuum packaging. Fabrication of LAPPDs is based largely on the application of thin-film materials to glass structures. In particular, a technique known as Atomic Layer Deposition (ALD) [7] enables the fabrication of large-area MCP amplification structures by conformally coating inactive, porous glass substrates [8, 9]. The technique is flexible as well as scalable, allowing for the independent optimization of the geometric, resistive, and secondary electron emission properties [8] of the channel plates.

In this paper, we present an analysis of the timing characteristics for $20 \mathrm{~cm} \mathrm{x}$ $2020 \mathrm{~cm} \mathrm{LAPPD}^{\mathrm{TM}}$ systems. At sufficient operational voltages, we observe singlephotoelectron time resolutions in the range of 50-60 picoseconds, consistent with those of commercial MCPs with comparable pore structures. Differential time resolutions are measured as low as 5.1 psec, with the large signal limit extrapolating below 2 picoseconds. Spatial resolutions are set by the granularity 25 of the economical stripline anode design (see Sec 2 ) and are measured to be less than $1 \mathrm{~mm}$ in both directions with respect to the stripline anodes. The median gain of the most recent MCP stack exceeds $10^{7}$.

\subsection{Structure of this paper}

Section 2 describes the essential elements of the LAPPD ${ }^{\mathrm{TM}}$ design. In Secso tion 3 we discuss the theoretical factors that determine the time resolutions of detectors generically, and MCP detectors such as LAPPDs specifically. We also identify the key observables and dependencies to be measured. Section 4 briefly describes the setup used to measure LAPPD timing, and Section 5 describes the measurement strategy. Section 6 describes the algorithms used to construct and fit the LAPPD pulses. Section 7 describes the results; conclusions are presented 

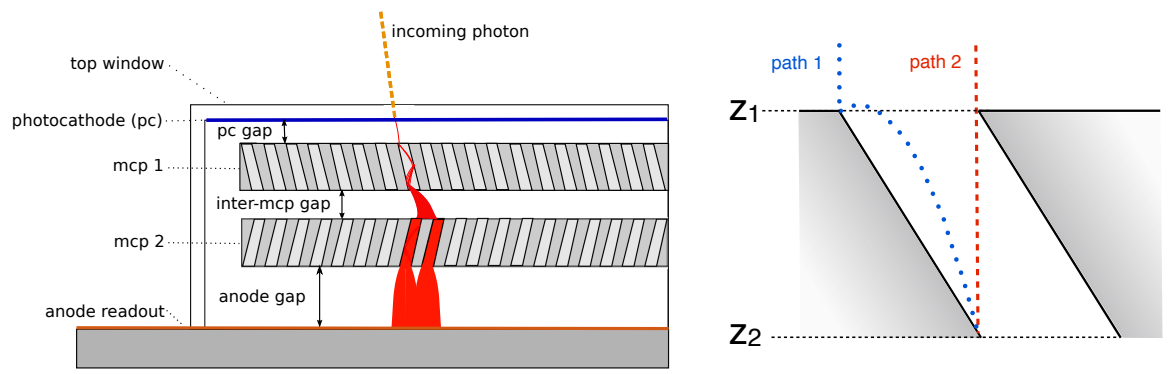

Figure 1: LEFT: The structure of an LAPPD ${ }^{\mathrm{TM}}$ photomultiplier tube. RIGHT: A schematic of photoelectrons entering the pore of an MCP. Both the dashed red and dotted blue trajectories reach height $z_{1}$ at the same time, but arrive at $z_{2}$ at different times due to different velocities and path lengths.

in Section 8

\section{Essential elements of the LAPPD ${ }^{\mathrm{TM}}$ design}

Figure 1 shows the structure of an $\operatorname{LAPPD}^{\mathrm{TM}}[10$. Light is incident on a photocathode, producing photoelectrons. These accelerate across a potential gap toward a pair of microchannel plates, which are high-gain structures consisting of thin plates with high secondary electron emission (SEE) enhanced, microscopic pores [1. Voltages of roughly $1 \mathrm{kV}$ are applied across each plate. Pores are oriented at $18^{\circ}$ bias angles in opposite directions. This prevents positive ions, produced by the electron cascade in the lower plate, from reaching and damaging the photocathode. It also provides a well-defined first strike for incoming electrons. Each electron entering a pore accelerates and strikes the pore walls, starting an avalanche of secondary electrons. The avalanche builds until the amplified pulse exits the bottom of the second MCP. This electrical signal is collected on an anode structure and passed through the vacuum assembly to sampling front-end electronics, which digitize the signal at 10-15 Gsamples/second. Spacing between the MCPs is set by glass grid spacers for the data reported here [10].

Anode coverage over large areas is achieved using a $50 \Omega$ micro-stripline design [11]. The positions of photon strikes on the photocathode are determined 
(i) by differential timing along the striplines, and (ii) by calculating a weighted centroid of the charge on adjacent striplines in the tranverse direction. This design allows economical area coverage as the number of readout channels scales linearly with length, rather than quadratically.

\section{Factors that limit and determine time resolution}

The timing characteristics of these photodetectors are determined by two key aspects of the detection process:

- Jitter in the formation of avalanches within the gain stage: This is determined by the physical properties of the MCP stack, such as pore diameters and bias angles, operational voltages, spacings between the components, and SEE characteristics.

- Information loss in the transmission and recording of the signal: This includes noise, attenuation of high frequency components as the pulse travels along the striplines, and quantization effects from pulse digitization.

\subsection{Jitter in the MCP signal formation, with respect to photon arrival}

70 in the transit of the initial photoelectron $(\mathrm{PE})$ and in the evolution of the avalanche. These fluctuations introduce a jitter in the start time $\left(t_{0}\right)$ and development of the MCP pulse with respect to the incoming photon. This jitter is largest for single-photoelectron pulses, independent of signal processing considerations. In the limit of many photoelectrons, it should decrease statistically 1

The most significant factor driving single-PE jitter is the "first strike". This is illustrated schematically in Fig 1, on the right. The dotted blue and dashed

\footnotetext{
1 The exact relationship between time resolution and $N_{\text {phot }}$ is complicated, depending on whether the photoelectrons enter one or several MCP pores, and whether the avalanche ultimately saturates.
} 
red photoelectrons accelerate across the photocathode gap, typically a few hundred volts. Both PEs reach the top plate $\left(z_{1}\right)$ at the same time with the same energy. The PE on the dotted blue trajectory immediately strikes the pore, while the dashed red trajectory continues deeper into the pore. A secondary electron produced at the strike-point of the dotted blue trajectory starts with $\mathrm{O}(1) \mathrm{eV}$ initial energy before accelerating towards $z_{2}$. The original photoelectron on the dahsed red trajectory accelerates towards $z_{2}$ over a shorter path, and starting with $\mathrm{O}(100) \mathrm{eV}$ energy. Thus, the dashed red trajectory arrives at position $z_{2}$ before the dotted blue one. The difference is $\mathrm{O}(10)$ picoseconds for these two strike points. There are many more possible trajectories for secondary electrons produced along path 1 , and there are many different first strike points within the pore. Given the current $20 \mu \mathrm{m}$ diameter and $8^{\circ}$ bias of the default $\mathrm{LAPPD}^{\mathrm{TM}}$ pores, these variations in trajectory lead to an $\mathrm{O}(10)$ picosecond jitter in $t_{0}$ of the avalanche. However, this jitter can be reduced by shrinking the pore size. Excellent single-PE time resolutions have been achieved using MCPs with pore diameters below $10 \mu \mathrm{m}[12$.

The number of secondaries and the randomization of their initial directions and energies further contribute to fluctuations in the development of the avalanche. The larger $N_{\text {secondaries }}$, the more these fluctuations will average out, and the more each individual pulse will behave in accordance with the mean behavior. A key way to reduce this is to increase the photocathode gap energy so that the first strike produces a large number of secondaries and to coat the pore surface with materials optimized for high secondary electron emission [8, 13.

In addition to variability from the first strike, some jitter in the time evolution of the avalanche is driven by the transition between the two MCPs of the gain stage. The avalanche from the first MCP will spread into a finite number of pores in the second-stage MCP. Depending on which pore in the first MCP is struck, fluctuations in this charge spreading will affect both the saturation and the timing of the resulting pulse. 

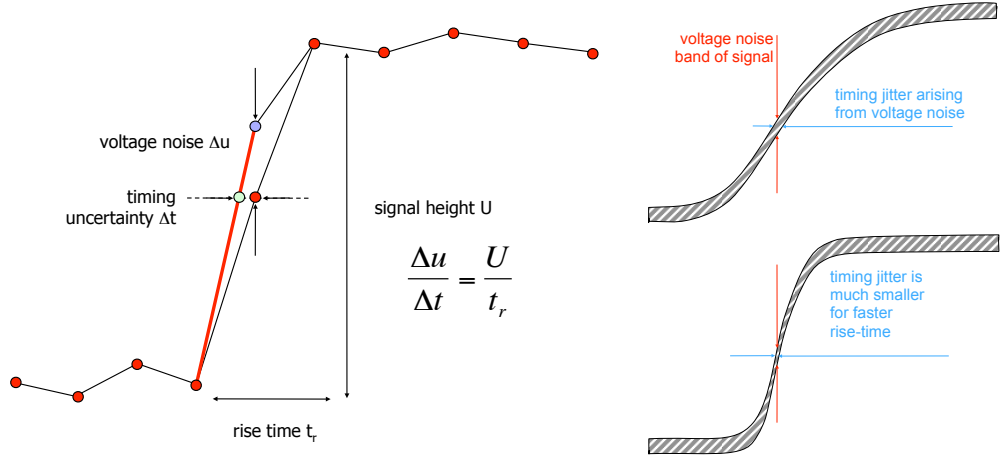

Figure 2: LEFT: an illustration showing how voltage noise translates into timing uncertainty for determining the time a signal crosses a threshold. RIGHT: two sketches showing how the impact of noise on timing also depends on the risetime of the signal.

\subsection{Uncertainties in extracting the arrival time from the $M C P$ signal}

Even if an MCP provided a precisely repeatable signal with fixed $t_{0}$, there would still be uncertainty in the arrival of that MCP signal due to limitations on extracting the signal from noise. Here we briefly discuss these issues, based largely on material from Ref [14, 15, 16.

Figure 2 demonstrates how the presence of noise introduces an uncertainty in the threshold crossing time of an otherwise repeatable signal. The right two plots in Fig 2 show the dependence of the size of the timing uncertainty for a given noise level on the rise time of the signal, which defines the slope between voltage and time.

By sampling more points along the rising edge, the uncertainty in the timing of the signal goes down with $\sqrt{N}$, assuming that the Nyquist-Shannon condition is met. Then, the time resolution can be described by Ritt's parameterization [15, 16, shown in Equation 1.

$$
\Delta t=\frac{\Delta u}{U} \times \frac{t_{\text {rise }}}{\sqrt{n}}=\frac{\Delta u}{U} \times \frac{t_{\text {rise }}}{\sqrt{f_{s} \times t_{\text {rise }}}}=\frac{\Delta u}{U} \times \frac{\sqrt{t_{\text {rise }}}}{\sqrt{f_{s}}}=\frac{\Delta u}{U} \times \frac{1}{\sqrt{3 f_{s} \times f_{3 d B}}}
$$

Where $\frac{\Delta u}{U}$ is the noise-over-signal, $t_{\text {rise }}$ is the rise-time of the signal, and $\mathrm{n}$ is the number of samples taken along the rising edge of the signals, $f_{s}$ is the 
samplng rate, and $f_{3 d B}$ is the highest frequency component of the pulse. The noise is assumed to be uncorrelated white noise, an assumption that might not be generically true in real life experiments.

The noise of the system is determined primarily by the design of the readout, particularly the electronics. The 15 Gsample/sec PSEC4 chip developed for $\mathrm{LAPPD}^{\mathrm{TM}}$ readout has a noise per channel of $700 \mu \mathrm{V}$ [17]. The oscilloscope measurements presented in this paper have noise levels around $3 \mathrm{mV}$. With

gains above $10^{7}$, the $\mathrm{LAPPD}^{\mathrm{TM}}$ system is capable of achieving noise-over-signal values well below 0.001 .

The rise time of the pulses is determined by the geometry and operational parameters of the MCPs, as well as the intrinsic analog bandwidth of the anode and front-end. Figure 3 shows that the highest frequency components of the signals above noise $(\sim 500 \mathrm{MHz})$ are well below the analog bandwidth allowed by the anode design and readout $(1.6 \mathrm{GHz}$ for the PSEC4 electronics and $3 \mathrm{GHz}$ for the scope) [11.

Based on these detector parameters and using Eq 1, we estimate that the $\mathrm{LAPPD}^{\mathrm{TM}}$ readout should be capable of time resolutions approaching a single picosecond. For single-photoelectrons, the overall time resolution is dominated by the $\mathrm{O}(10)$ psec intrinsic jitter from the MCPs, rather than the readout. In the limit of many photoelectrons, and for future designs with optimized MCPs, improvements in time resolution will come from improvements to the readout such as higher bandwidth anodes, faster sampling rates, and lower noise electronics.

\subsection{Relevant observables}

The timing limitations imposed by the properties of the gain stage can be measured by studying the absolute timing of the detector in single-PE operation with repect to a photodiode triggered by the laser (see Section 4). It can be characterized by the width of the Transit Time Spread (TTS). The limiting factor for multiple photoelectron operation is driven by the noise over signal $(\mathrm{N} / \mathrm{S})$ quality of the readout and can be measured using differential timing: the 


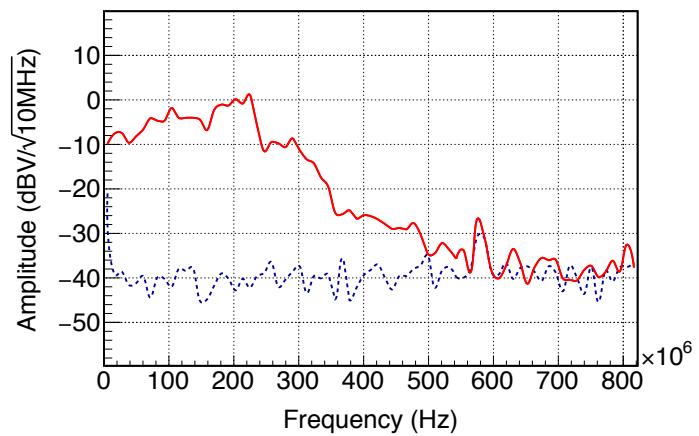

Figure 3: Frequency spectrum of the MCP signal plus noise (solid red) overlaid on the pure noise spectrum (dashed blue). Signal dominates over noise only up to around $500 \mathrm{MHz}$.

difference in arrival times of the two pulses at opposite ends of the delay line anode. In differential timing measurements, fluctuations in the start and development of the signal cancel out, leaving only the uncertainties due to noise in the readout. This observable we will refer to as the Differential Time Spread (DTS), and it improves with increasing signal size (or decreasing noise), regardless of whether the large signal is achieved by more photoelectrons or higher gains.

The timing characteristics of LAPPD ${ }^{\mathrm{TM}}$ systems will be relevant to different applications in different ways. Looking at some examples from high energy physics, water Cherenkov (WCh) detectors are single-photon counting devices; events are reconstructed using precision measurements of each individual emitted photon. The timing requirements are less demanding $(<100 \mathrm{psec})$, but the timing capabilities are more limited due to the intrinsic jitter of the gain stage on the single-PE TTS. Time-of-flight (TOF) detectors require much better time resolutions, but also look at much larger signals - typically 50 photoelectrons produced by a high energy particle traversing a radiator [14]. For these applications we are more concerned with the large-signal limits of LAPPD ${ }^{\mathrm{TM}}$ systems, determined by the readout. These large-signal resolutions can be characterized by looking at the DTS. In this paper we will discuss both the TTS and DTS, as 
well as their dependence on operational parameters such as voltage and S/N.

\section{Experimental setup}

In this section we will briefly describe the test setup used to characterize

$\mathrm{LAPPD}^{\mathrm{TM}}$ detector systems. More detailed discussions of the characterization facility can be found in Ref $[18$.

For all of the measurements, signals are produced by shining UV light from a sub-picosecond pulsed Ti::Sapphire laser on a thin aluminum photocathode 2 $\mathrm{mm}$ above the gain stage. The arrival time of the MCP pulses can therefore be measured precisely against a trigger signal derived from these laser pulses. Using a statistical technique described in Sec 5. we can achieve low light intensities and study the characteristics of MCP pulses derived from single-photoelectrons. The laser spot size is roughly $1 \mathrm{~mm}$, and a motorized stage is used to study the response and different locations on the detector surface. The shapes of the pulses are measured using a fast oscilloscope and written to disk for offline analysis.

This paper presents the results of two separate studies. One set of measurements was performed on an unsealed LAPPD ${ }^{\mathrm{TM}}$ detector system inserted into a larger, steel vacuum chamber (the "8-inch Chamber") [18. The other study was performed on a vacuum tight, resealable glass LAPPD ${ }^{\mathrm{TM}}$ detector, known as the "Demountable" [18.

\subsection{The 8" chamber}

Early studies of 8" microchannel plates were performed before the glass vacuum packaging for LAPPDs was developed. A complete detector stack was assembled with proper electrical contacts, and inserted into a larger, stainless steel vacuum chamber (Fig 4). Laser light entered the chamber through a quartz window and was directed onto the MCP stack using mirrors. Signals were brought out of the chamber through SMA feedthroughs. Due to the limited number of feedthroughs, only four striplines could be instrumented at a time. One key advantage of the 8 " chamber was the ability to independently control the voltages at each stage of the MCP stack. 

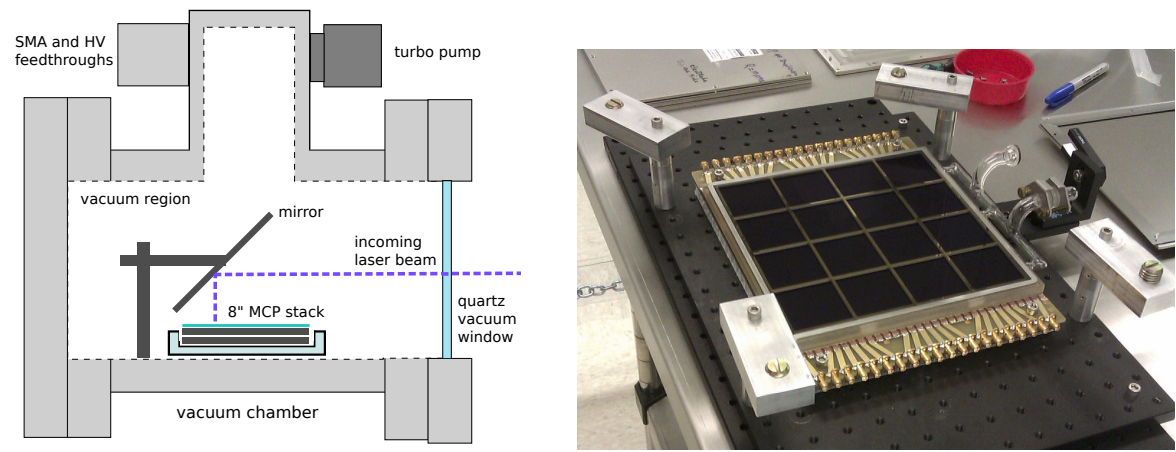

Figure 4: LEFT: A schamatic illustration of the 8" MCP test-chamber. RIGHT: A picture of the assembled demountable LAPPD before placing and sealing the top window.

\subsection{The Demountable detector}

The Demountable assembly is an 8.66 " x 8.66 " glass vacuum tube detector made from LAPPD ${ }^{\mathrm{TM}}$ production parts, whose differences from the sealed detector of the design goal are that: 1) the tube is actively pumped rather than hermetically sealed; 2) the seal between the fused silica top window and the tube body is with an O-ring rather than an indium seal; and 3) the photocathode is a thin aluminum layer rather than a bialkalai film, as the Demountable is assembled in air. Inside the demountable we place a stack of 2 ALD-functionalized 8 "x8" MCPs, with spacers in the three gaps: a) between the MCP stack and the anode; b) between the two MCPs; c) between the MCP stack and the photocathode [10]. The spacers are ALD-coated with a resistive layer. The resistances of the spacers and plates set the respective operational voltages, and allow signals to pass from the photocathode through the stack to the anode strips, which are DC terminated with $10 \mathrm{k} \Omega$ resistors [10]. Thus, unlike the 8" chamber, this voltage divider does not allow independent voltage control over each component. High voltage electrical contact is made by connecting to the aluminum side of the top-window on end-tabs where the window extends past the vacuum region of the Demountable body. 


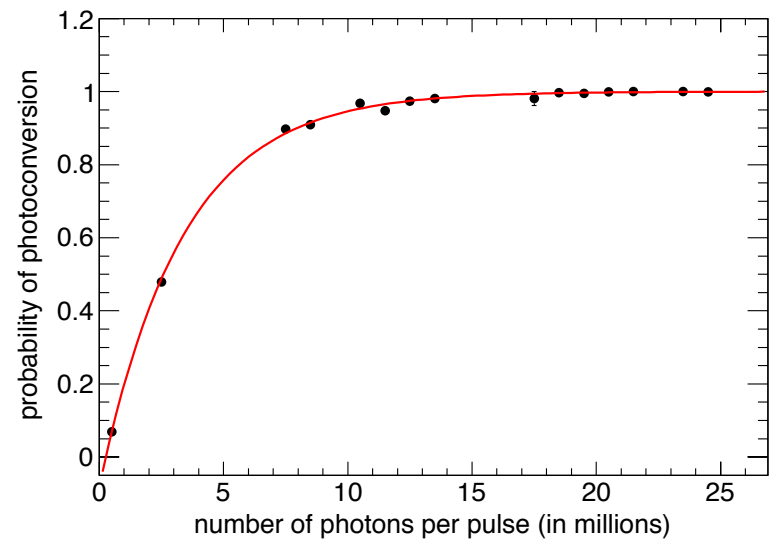

Figure 5: Probability of the Ti::Sapphire laser pulse generating an MCP signal on the aluminum photocathode, plotted as a function of pulse energy. At sufficiently high laser intensities, this probability approaches unity; as the laser is attenuated below roughly 10 million UV photons per pulse, the fraction of laser pulses producing an MCP signal begins to drop and eventually approaches zero.

\section{Measurement strategy}

A pulsed, sub-psec laser was used to characterize the time resolution of the large-area MCPs. Absolute timing of the MCP response was measured in relation to a fast photodiode triggered by the laser. Single-photoelectron operation was achieved by controlling photon statistics.

The average UV laser power, of order 100 nano-Watts, was sufficient to produce many photoelectrons per pulse, even with a low quantum efficiency (QE) aluminum photocathode. Without attenuation, the fraction of laser pulses with an observed MCP signal was $100 \%$. However, the beam could be attenuated to the point where some fraction of laser pulses produced no discernible signal, as determined from the oscilloscope data using analysis techniques described in Section 6.1. Once the detector was operating in a regime where the fraction of events with good pulses was sufficiently low, the probability of producing more than one PE was statistically suppressed. Figure 5 shows the relationship between average UV intensity and the probability of an MCP signal. The slope of 


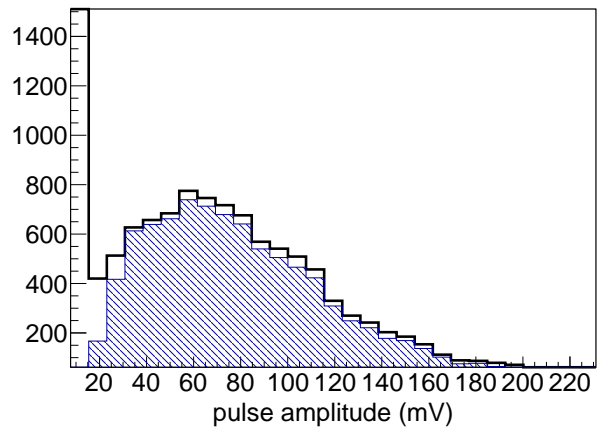

Figure 6: The amplitude distribution of pulses from the 8" chamber (in thick black), showing the separation between the peak of the distribution and the pedestal near zero. The shaded blue region shows the same distribution after applying the TOT cut and requiring only one pulse.

this plot at low laser intensities can be used to extrapolate to higher intensities, providing a statistical handle on the number of photoelectrons. This approach does not depend on the choice of photocathode or precise knowledge of the QE.

\section{Analysis techniques}

\subsection{Pulse selection}

Pulses are separated from background on the basis of a time over threshold

(TOT) cut. Pulses are identified as a region of the signal trace where the charge exceeds a predetermined threshold of $5 \mathrm{mV}$ for a duration longer than $1.0 \mathrm{nsec}$. We explored several possible pulse selection methods, but found this technique to be simple, robust, and effective at separating signals from white noise and RF interference from the laser. The result of this cut on the amplitude distribution of the MCP pulses is show in Fig 6. This analysis requires events where only one pulse is identified, although the number of events with $N_{\text {pulses }}>1$ is negligible.

\subsection{Timing algorithms}

Several time reconstruction algorithms were tested : constant-fraction discrimination, fitting with a Gaussian distribution, and template fitting. These 
A simple, but nonetheless effective technique for reconstructing pulse arrival times is to use a Constant-Fraction Discriminator (CFD). This technique is robust and simple, but does not capture the full information contained in the pulse shape, and it suffers from a time slewing for pulses of different shapes.

255 distribution. As illustrated by Fig 7, pulse shapes from the LAPPDs are nonGaussian. Any naive fit of a Gaussian distribution to these pulses will yield worse transit time spreads than simple CFD methods. However, the pulses can be well approximated by a Gaussian function from the rising edge to a point, slightly past the peak of the pulse.

This method is implemented in two steps. First, we fit the pulse peak with a Gaussian to determine the peak time. Next, using the fitted peak as a reference, we define a fit range and apply a Gaussian fit along the rising edge. This give results close to those of the template fit but is less robust, with a higher failure rate for slightly misshapen pulses.

The most robust method for extracting pulse arrival times is to fit the MCP pulses with a data-derived template waveform. Templates are created by selecting pulses, using a stringent pulse quality cut, and averaging them together. In some instances this process was repeated a second time, where the fitted time using the original template was used to line the pulses up before combining them to create a more narrow pulse shape. Since the TTS of the pulses $(<100$ psec $)$ is considerably smaller than the typical pulse width $(\mathrm{FWHM}<1 \mathrm{nsec})$, iterative template-making does not substantially improve the results. Once the template has been created, it is used to fit individual pulses. Pulses are interpolated and 275 over a predetermined range of the template. Figure 8 shows an example pulse with the result of the template fit overlaid. 

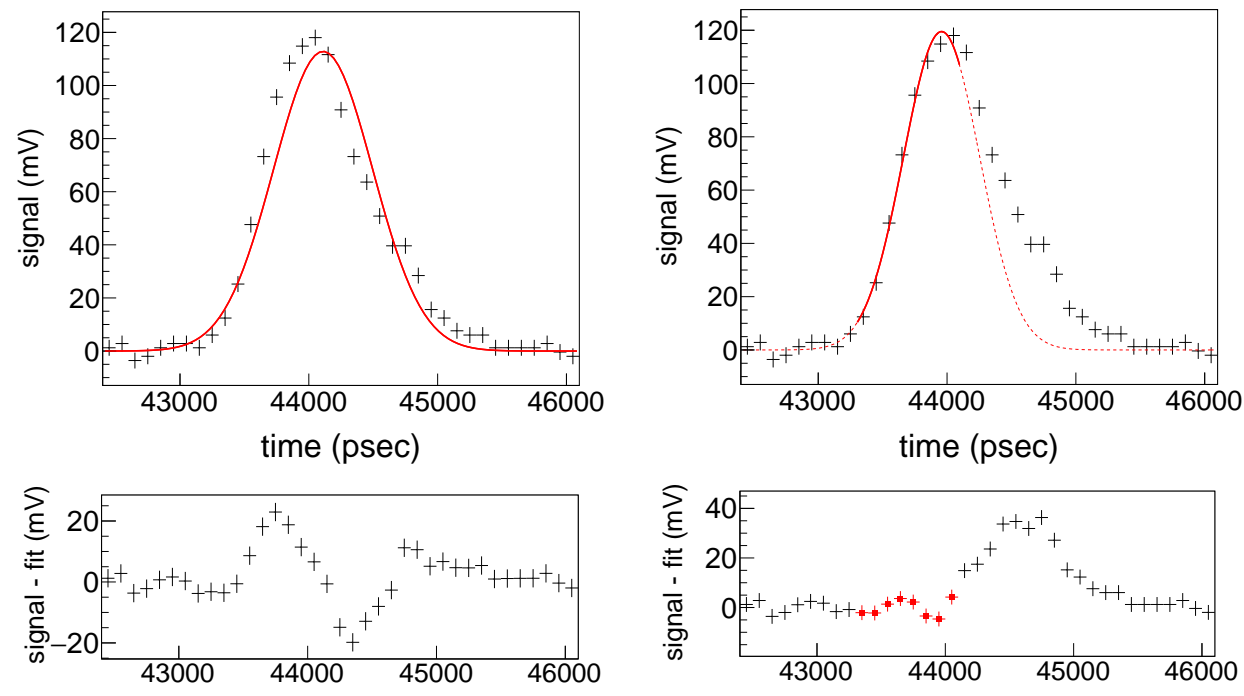

Figure 7: Two examples of Gaussian fits to an MCP pulse, plotted above a graph showing the difference between the fitted curve and data points. The error bars correspond to $3 \mathrm{mV}$ white noise on the oscilloscope. On the left plot, the pulse is fitted over the full range. The right plot shows the prescription used in the data analysis of fitting the rising edge only. The solid line and closed circles on the right correspond to the region used in the fit. The dashed line and open points are included to show the fit beyond the range of interest. 

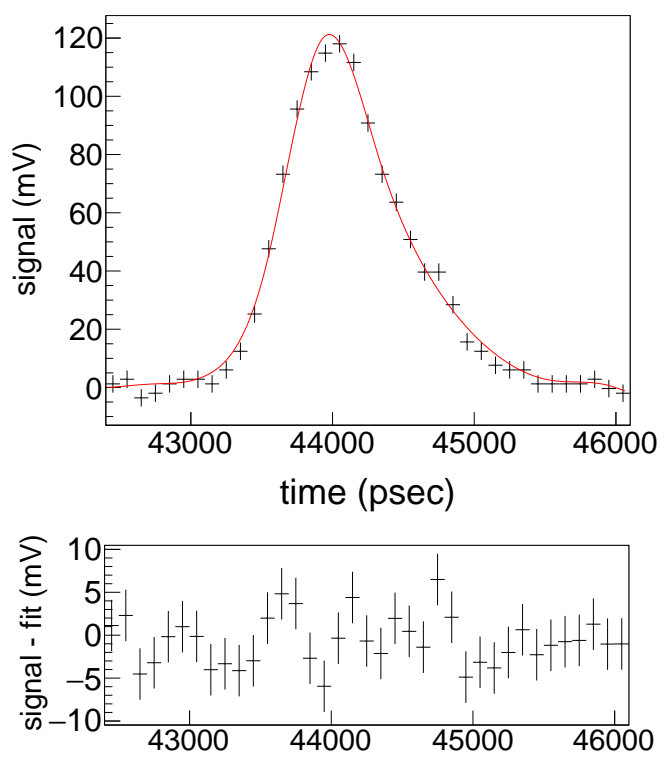

Figure 8: Best fit of the template shape to an example pulse, plotted over the full range of the pulse. The lower plot shows the difference between each data point and the fitted curve, with $3 \mathrm{mV}$ error bars corresponding to oscilloscope noise, over the range of interest. 


\section{Results}

\subsection{The single-photoelectron response}

to its nearest neighbor. We found that fitting the arrival time on the dominant stripline alone was adequate to obtain single-PE resolutions consistently below 

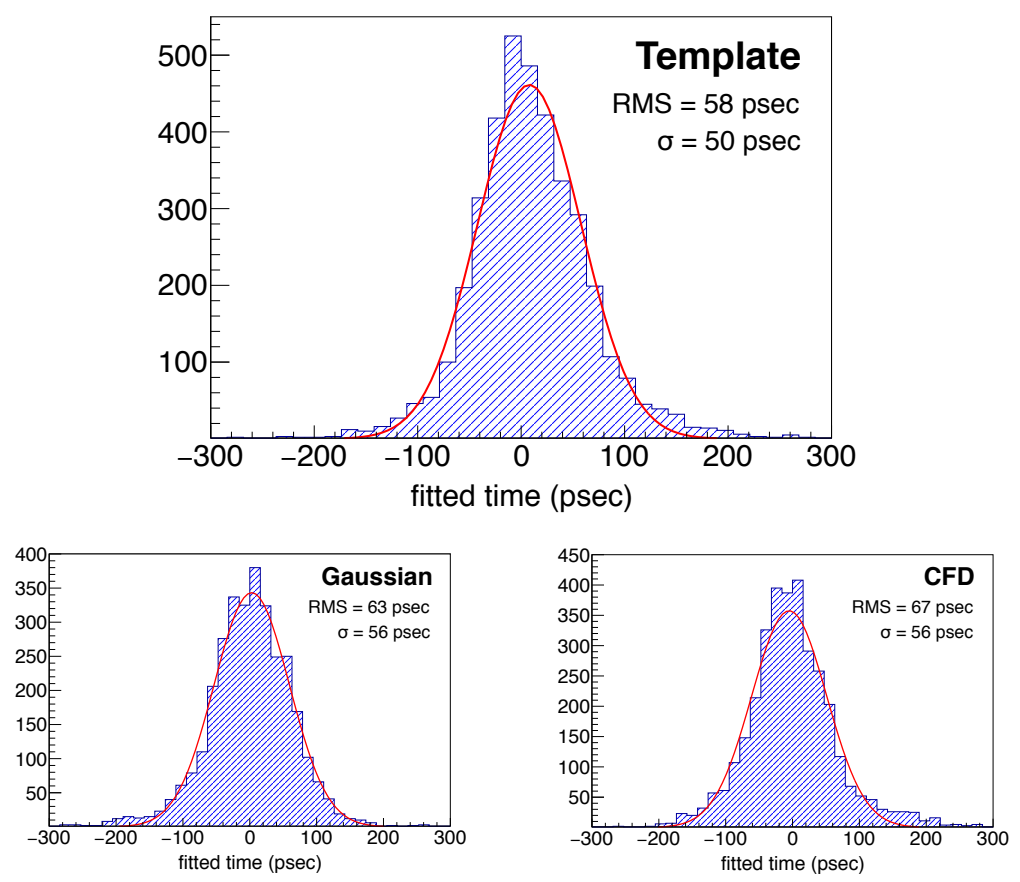

Figure 9: The single-PE transit-time spread measured for the LAPPD ${ }^{\mathrm{TM}}$ stack inside the 8" chamber, derived using the three different fit methods (see text). TOP: Using the template fit method. The RMS of the distribution is 58 psec; The sigma of the fitted gaussian is 50 psec. Numerically integrating the distribution, one finds that $68 \%$ of all events fall within \pm 47 psec. LOWER LEFT: Using the gaussian fit method. The RMS of the arrival times is 63 psec, and the fitted sigma is 56 psec. LOWER RIGHT: Using the CFD algorithm. The RMS of the arrival times is $67 \mathrm{psec}$, and the fitted sigma is 56 psec. Given the inherent limitations of these fit methods, the non-Gaussian character of the reconstructed TTS and wider tails compared to the template fit are expected. 

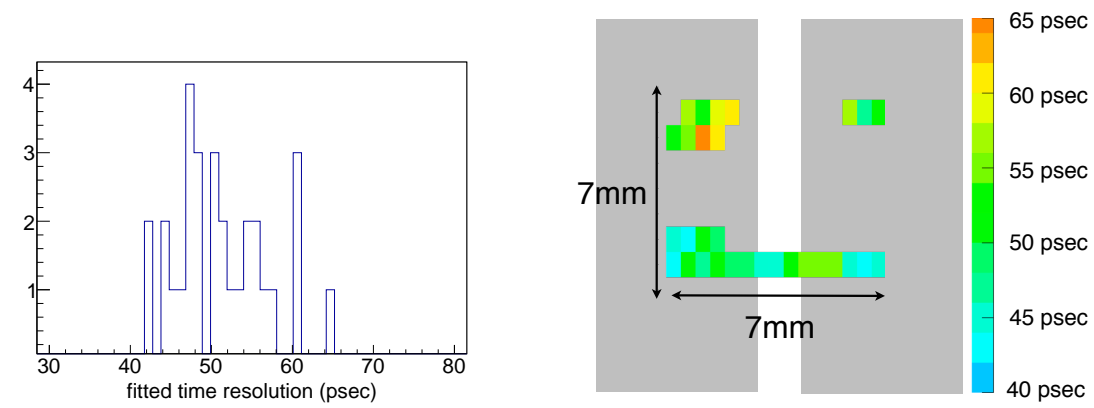

Figure 10: LEFT: A histogram of the fitted sigma of the TTS measured at 30 points over a $7 \mathrm{~mm}$ by $7 \mathrm{~mm}$ square. The mean value of the fitted time resolution is 51 picoseconds, and the RMS from measurement to measurement is 6 picoseconds. RIGHT: A plot showing the locations and values of each measurement, relative to the stripline pattern. The color scale represents the range of measured time resolutions (color online).

65 picoseconds, even in the region where signal is split between the two striplines (Fig 11).

The width of the TTS depends on operational voltages, particularly across the gaps between the various stages of the MCP chain. As the gap voltages drop below critical values, the time resolution resolution degrades. Figure 12 shows how the shape of the MCP pulses in the 8" chamber depend on changes to the three voltages across the gaps in the MCP stack. The most dramatic effects were seen from changes in the voltage across the gap between the two MCPs. Figure 13 shows the fitted sigma of the TTS as a function of the potential difference across the anode gap, inter-MCP gap, and photocathode gap. Optimal timing was achieved with all of these voltages set above 200-300 Volts. The dependence was strongest for the inter-MCP gap and photocathode gap, consistent with the observation that this gap had the largest impact on pulse shape.

Similar relationships between gap voltages and time resolution were observed in the Demountable detector. In the Demountable, we did not originally have access to grid spacers with resistances well matched to those of the MCPs. 325 Consequently, the voltages across all three gaps were below $100 \mathrm{~V}$ when the 

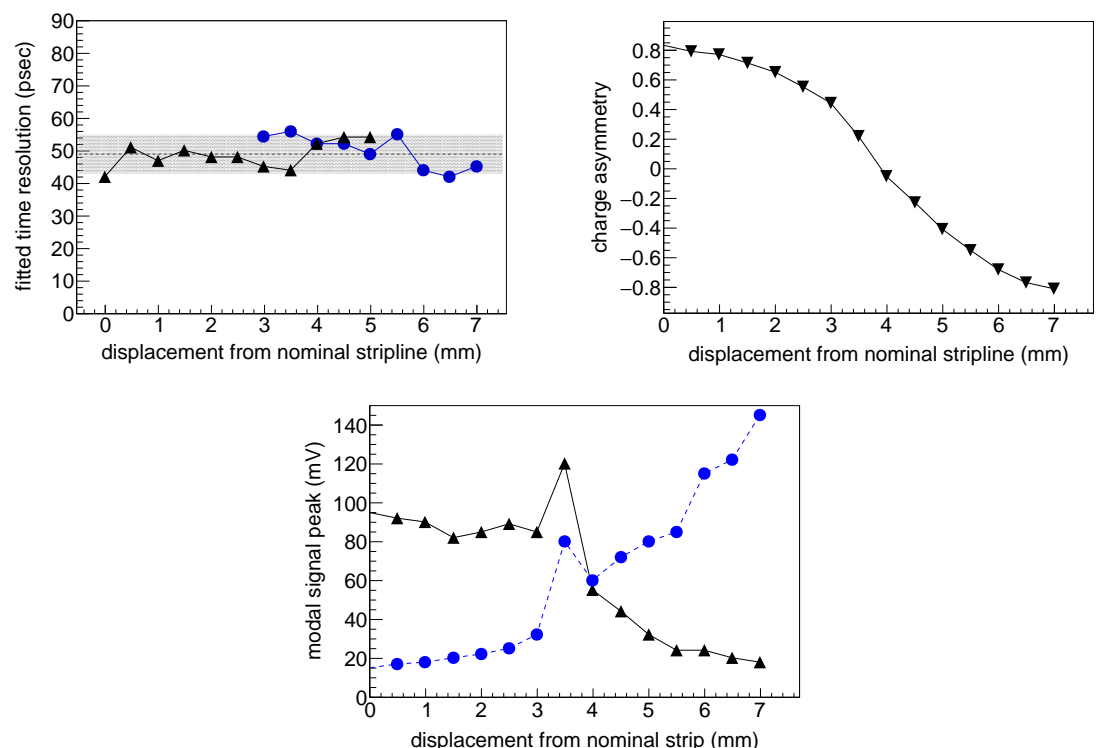

Figure 11: UPPER LEFT: The fitted time resolution as a function of approximate displacement, measured on the nominal stripline (black triangles) and the neighboring stripline (blue circles). The dashed line and shaded band indicate the mean and RMS of the measured resolutions over the scan. UPPER RIGHT: The asymmetry in charge measured on the two neighboring striplines, plotted as a function of approximate displacement from the nominal strip. An asymmetry of 1 means that all of the charge is on the nominal stripline and -1 means all of the charge on the neighbor. BOTTOM: The modal signal peak, as a function of displacement from the nominal strip. Black triangles correspond to the peak signal on the nominal stripline and blue circles, the neighboring strip. The spike in signal size at displacement $\sim 3.5 \mathrm{~mm}$ is not understood, but the fractional signal on each stripline is consistent with the location of the beam. 

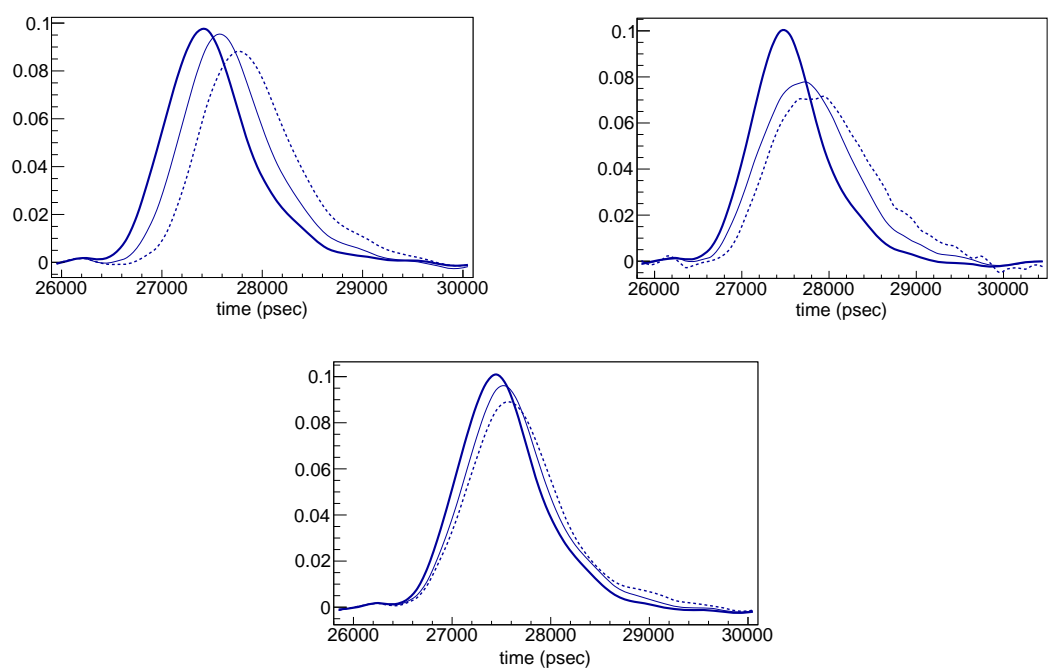

Figure 12: UPPER LEFT: Average pulse shape in the 8" chamber for three different photcathode voltages: $500 \mathrm{~V}$ (thick line), $200 \mathrm{~V}$ (thin line), $100 \mathrm{~V}$ (dashed), with $1 \mathrm{kV}$ across each MCP, $500 \mathrm{~V}$ across the inter-MCP gap, and $400 \mathrm{~V}$ accross the anode. UPPER RIGHT: Average pulse shape for $500 \mathrm{~V}$ (thick line), $200 \mathrm{~V}$ (thin), and $100 \mathrm{~V}$ (dashed) across the inter-MCP gap, with $400 \mathrm{~V}$ across the anode and photocathode. LOWER LEFT: Average pulse shape for $500 \mathrm{~V}$ (thick line), $200 \mathrm{~V}$ (thin), and $100 \mathrm{~V}$ (dashed) across the anode gap, with $400 \mathrm{~V}$ across the photocathode and $500 \mathrm{~V}$ across the inter-MCP gap.

MCPs were operating at full voltage. In a later study, these spacers were replaced with a higher resistance set. Figure 14 shows the average pulse shape for the demountable with the low resistance and high resistance grid spacers, along side the corresponding TTS distributions. Consistent with observations in the 8" chamber, the Demountable with low voltages across the gaps delivered time resolutions above $100 \mathrm{psec}$, while later studies with higher gap voltages demonstrated resolutions below 80 psec.

Further improvements in LAPPD ${ }^{\mathrm{TM}}$ single-PE time resolution may be achieved through reductions in pore size [19], and higher yield of the first strike in the pore resulting from higher SEE surface coatings. 


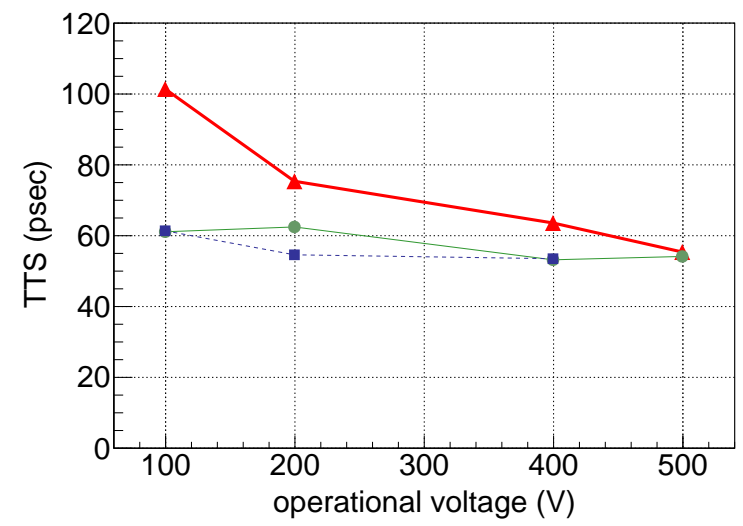

Figure 13: Transit-time spread of MCPs in the 8" chamber, plotted as a function of the key operational voltages: across the gap between the photocathode and top of the first MCP (green circles), between the two MCPs (red triangles), and between the bottom of the second MCP and anode (blue squares). Timing for all gap voltages is preferred to be above $200 \mathrm{~V}$, and performance is most sensitive to the photocathode and inter-MCP gaps.
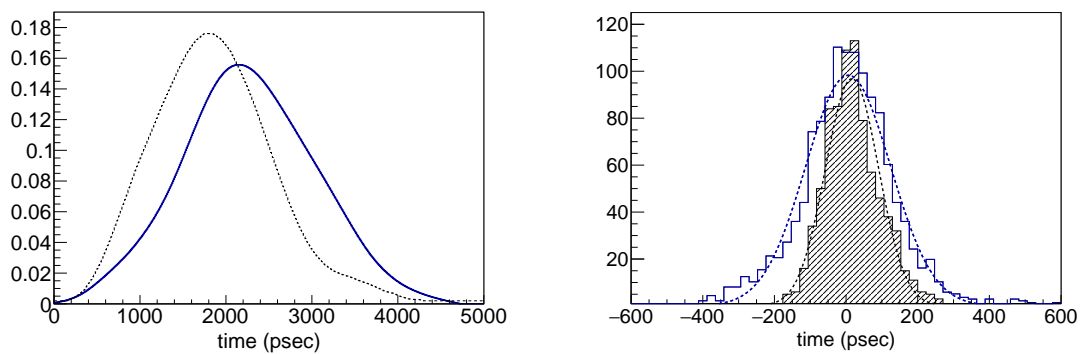

Figure 14: LEFT: Average pulse shape for 2 different configurations of the demountable $\mathrm{LAPPD}^{\mathrm{TM}}$ : one with low voltages $(<100 \mathrm{~V})$ across all of the gaps (blue solid line), and one with higher gap voltages (dashed black). RIGHT: The TTS for the two configurations. The high gap-voltage configuration is the shaded black histogram, with $\sigma=78$ picoseconds for the fitted Gaussian (dashed line). The TTS for configuration with low gap voltages is shown by the blue solid line, with a fitted $\sigma$ of 128 psec. 


\subsection{Differential timing and the large-signal limit}

Even for single-photoelectrons, the pulse-by-pulse jitter cancels out when one looks at the differential time resolution - jitter in the difference between the arrival of the signal at the two ends of the anode. Differential timing resolution

340 is not limited by the intrinsic properties of the gain stage except inasmuch as they provide large signal. Rather, differential time resolution is limited by the characteristics of the pulse such as the rising edge and size of the pulse, and electronics characteristics such as noise-over-signal (N/S), analog bandwidth, and sampling rate.

The Differential Time Spread (DTS) thus provides a handle on the time resolving characteristics of the detector system, minus those of the MCP stack. It indicates the limiting TOF-resolution for large, multi-photoelectron pulses.

Figure 15 shows the differential time resolution for single-photoelectrons in the Demountable LAPPD ${ }^{\mathrm{TM}}$, operating at $2.7 \mathrm{kV}$, which corresponds roughly $1.25 \mathrm{kV}$ per MCP. The RMS of the distribution is 6.8 psec. The sigma of the fitted gaussian is $5.1 \mathrm{psec}$, although the shape of the distribution is nongaussian. The bell-bottomed shape of the DTS distribution is expected since it is effectively the sum of multiple Gaussians, each corresponding to the resolution for a particular range of pulse sizes. Small pulses have a broader Gaussian, while large pulses are more narrow. Even so, by simply cutting more tightly on the quality of the $\chi^{2}$ of the template fit we achieve a more Gaussian subset of $66 \%$ of the original DTS distribution with an RMS of 4.8 psec and fitted Gaussian of 4.3 psec (shown on the right in Fig 15). Some of this limiting resolution is due to the finite spot size of the laser beam $(\sim 0.5 \mathrm{~mm})$, and limitations of the digital readout of the two independent oscilloscope channels used to measure each side of the stripline.

The data sets can be further divided into bins of N/S. Figure 16 shows the relationship. Even with single-PE data, N/S levels as low as 0.006 were achievable, due to the very high pulse amplitudes of the Demountable (see ${ }_{365}$ Fig 18 ). As expected, pulses with smaller N/S (corresponding to larger signals) demonstrate better time resolution. A linear fit to these points intersects with 

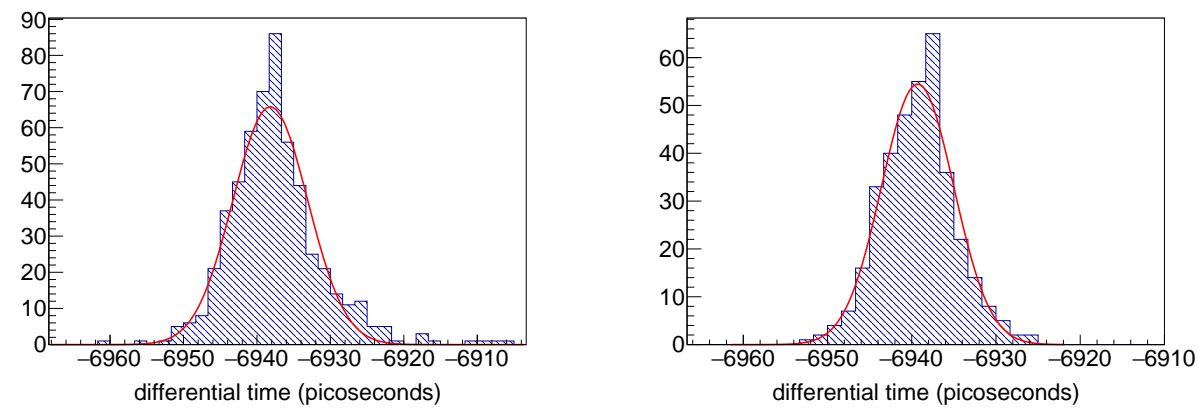

Figure 15: LEFT: Differential time spread for the demountable detector, fitted with a gaussian. The RMS of the distribution is 6.4 psec, and sigma of the gaussian is 5.1 psec. RIGHT: With a modest cut on $\mathrm{N} / \mathrm{S}<0.01$, the sample is reduced by only $44 \%$, leaving a DTS distribution with a more gaussian shape with RMS 4.8 psec and fitted sigma of 4.3 psec.

the y-axis at a resolution close to 1 picosecond for large signals. In contrast, the single-PE absolute time resolution is dominated by intrinsic jitter in the MCP stack and remains above 40 psec, even in the large-signal limit (Figure 17). The fluctuations in avalanche formation that dominate the single-PE TTS, cancel in the differential timing measurement. This explains the robustness of the relationship between the differential time resolution and $\mathrm{N} / \mathrm{S}$, measured with different MCP stacks and at different times.

The measured differential time resolution of 5.1 psec corresponds to a spatial resolution in the direction along the striplines of roughly $0.44 \mathrm{~mm}$. Figure 19 shows the average time delay between the two ends of a stripline as a function of position along that stripline. These data show a linear relationship with a slope of roughly $0.57 c$, in agreement with the measured speed of signal propagation along the micro-striplines [11.

\section{Conclusion}

LAPPD $^{\text {TM }}$ detector systems are capable of providing absolute time resolutions for single-PEs consistently below 100 picoseconds, and typically below 60 picoseconds. Moreover, the large gain and high signal-to-noise ratio make LAPPD pulses easy to separate from background. While absolute time resolu- 

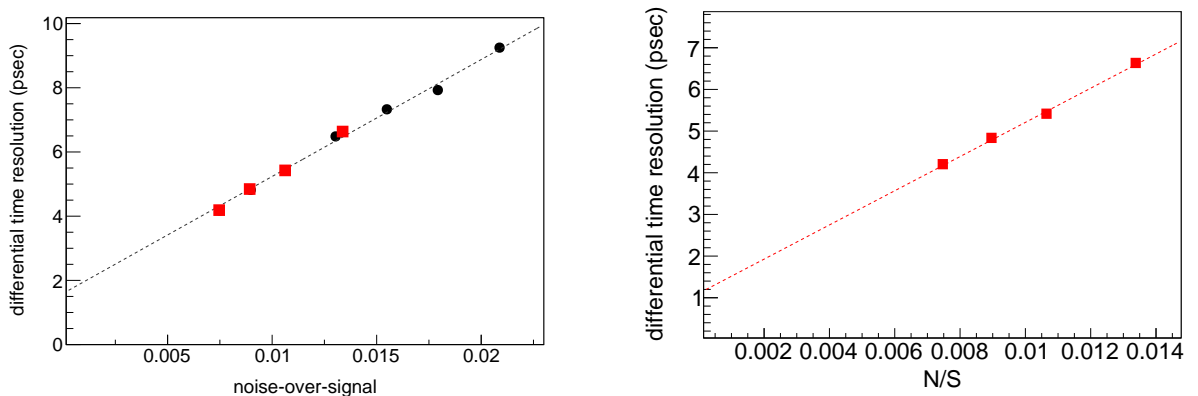

Figure 16: LEFT: Differential time resolution as a function of Noise/Signal, plotted for data collected using the Demountable detector in 2014 (red squares) and the 8" chamber in 2012 (black circles). Error bars are smaller than the marker size. The intercept of fitted line intersects at 1.6 psec. RIGHT: Same plot, for 2014 demountable data only. The intercept of the fit intersects with 1.1 psec.

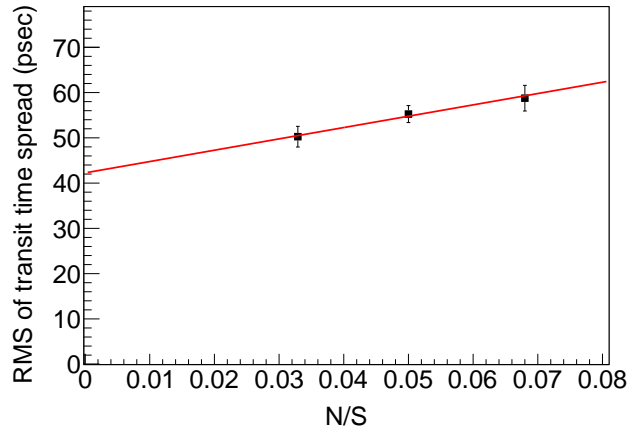

Figure 17: Single-photoelectron time resolution measured in the 8" chamber, binned by noise/signal ( $2 \sigma$ error bars). Slight improvements are seen with larger signals, but the singlePE resolutions are limited by fundamental constraints such as pore diameter and asymptotically approach 42 psec for large S/N. 

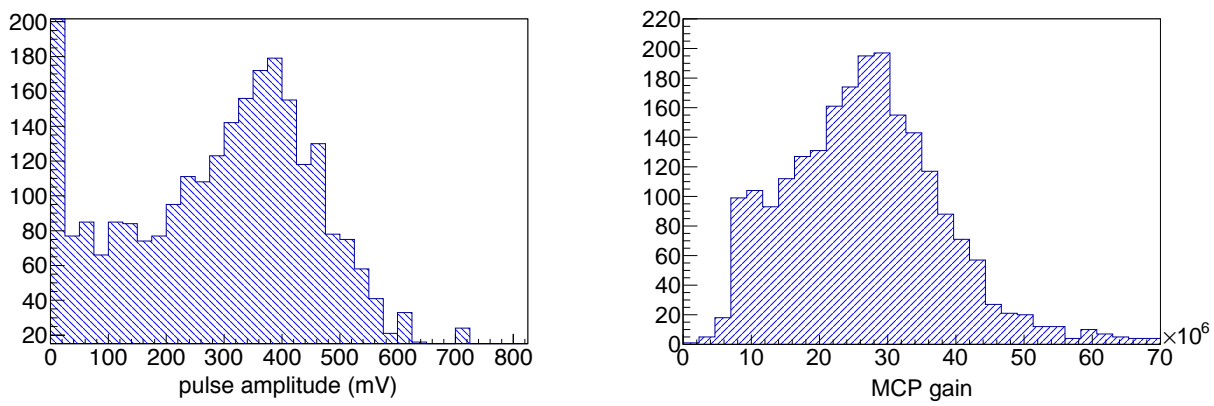

Figure 18: LEFT: Distribution of signal amplitudes from the demountable LAPPD ${ }^{\mathrm{TM}}$ operating at $2700 \mathrm{~V}$, including pedestal events (zero bin). RIGHT: The reconstructed MCP gain distribution for good pulses, with peak at around $3 \times 10^{7}$.
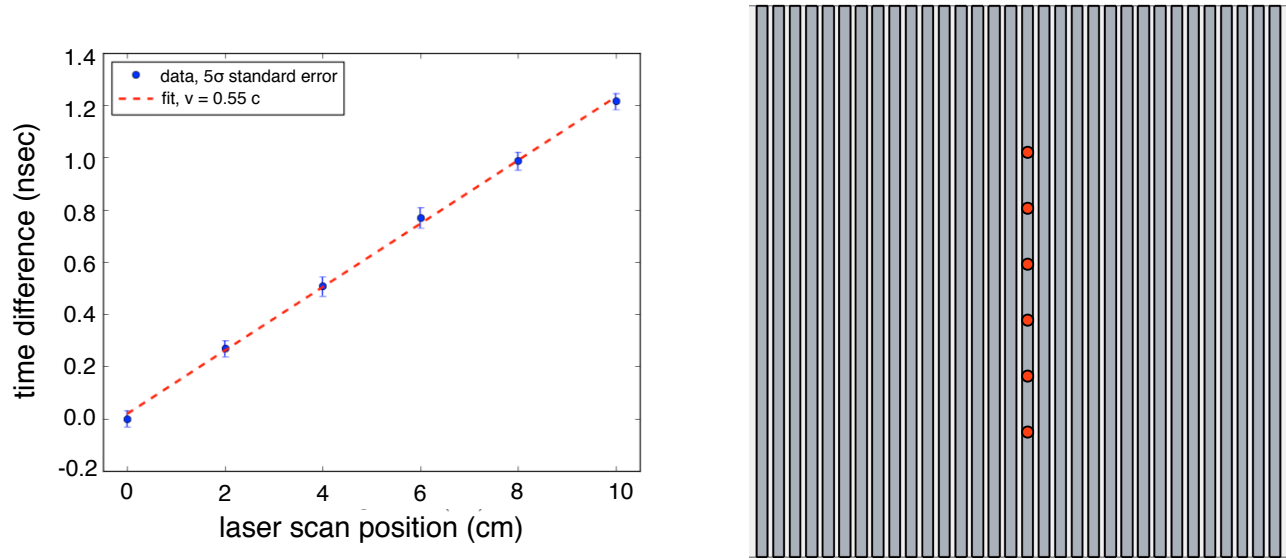

Figure 19: Mean differential time between the arrival of the pulse at the two ends of the microstripline anode, plotted as a function of position along the anode (left). 
single-PE and large signal performance.

\section{Acknowledgements}

This work could not have been done without the talent and dedication of 395 Engineering Center, Mary Heintz and Mark Zaskowski of the UC Electronics Development Group. We thank Harold Gibson and Haidan Wen of the ANL Advanced Photon Source for laser and electronics support at the APS testing lab. We thank Jeffrey Elam and Anil Mane (ANL), and Neal Sullivan (Arradiance)

for their critical role in providing the ALD functionalized MCPs used in this paper. We are grateful to Gary Drake for his help in mitigating the electronics noise and Dean Walters with advice on vacuum-related matters. We thank Jason McPhate and Oswald Siegmund for advice and encouragement. Finally, we would like to thank Incom Inc. for providing the 8" substrates, technical assistance with glass parts, and helpful discussions.

The activities at Argonne National Laboratory were supported by the U. S. Department of Energy, Office of Science, Office of Basic Energy Sciences and Office of High Energy Physics under contract DE-AC02-06CH11357, and at the University of Chicago by the Department of Energy under Contract DE410 SC-0008172 and the National Science Foundation under grant PHY-1066014. Matthew Wetstein is grateful for support from the Grainger Foundation. 


\section{References}

[1] J. L. Wiza, Microchannel plate detectors, Nucl. Instrum. Methods 162 (1979) 587-601.

[2] A. S. Tremsin, O. H. W. Siegmund, Spatial distribution of electron cloud footprints from microchannel plates: Measurements and modeling, Review of Scientific Instruments 70 (8) (1999) 3282-3288. doi:http://dx.doi. org/10.1063/1.1149905.

[3] J. S. Milnes, J. Howorth, Picosecond time response characteristics of microchannel plate pmt detectors, Proc. SPIE 5580 (2005) 730-740. doi: $10.1117 / 12.568180$

[4] M. Akatsu, Y. Enari, K. Hayasaka, T. Hokuue, T. Iijima, K. Inami, K. Itoh, Y. Kawakami, N. Kishimoto, T. Kubota, M. Kojima, Y. Kozakai, Y. Kuriyama, T. Matsuishi, Y. Miyabayashi, T. Ohshima, N. Sato, K. Senyo, A. Sugi, S. Tokuda, M. Tomita, H. Yanase, S. Yoshino, MCPPMT timing property for single photons, Nucl. Instrum. Methods A 528 (3) (2004) 763 - 775. doi:http://dx.doi.org/10.1016/j.nima.2004.04. 207.

[5] K. Inami, N. Kishimoto, Y. Enari, M. Nagamine, T. Ohshima, A 5 ps TOFcounter with an MCP?PMT, Nucl. Instrum. Methods At 560 (2) (2006) 303 - 308. doi:http://dx.doi.org/10.1016/j.nima.2006.01.027.

[6] O. H. W. Siegmund, Ch 7. amplifying and position sensitive detectors, Vacuum Ultraviolet Spectroscopy II. Series: Experimental Methods in the Physical Sciences 32 (1998) 139-175. doi:10.1016/S0076-695X(08) $60280-\mathrm{X}$

[7] S. M. George, Atomic layer deposition: an overview, Chemical reviews 110 (1) (2009) 111-131. doi:10.1021/cr900056b.

[8] S. J. Jokela, I. V. Veryovkin, A. V. Zinovev, J. W. Elam, Q. Peng, A. U. Mane, The Characterization Of Secondary Electron Emitters For Use In 
Large Area Photo-Detectors, Application of Accelerators in Research and Industry AIP Conf. Proc 1336 (2011) 208-212. doi:10.1063/1.3586090.

[9] N. Sullivan, P. de Rouffignac, D. Beaulieu, A. Tremsin, K. Saadatmand, D. Gorelikov, H. Klotzsch, K. Stenton, S. Bachman, R. Toomey, Novel microchannel plate device fabricated with atomic layer deposition, Proceedings of the Ninth International Conference on Atomic Layer Depositiondoi : 10.1016/0003-4916(63)90068-X.

[10] B. W. Adams, A. Elagin, J. W. Elam, H. J. Frisch, J.-F. Genat, J. S. Gregar, A. U. Mane, M. J. Minot, R. Northrop, R. Obaid, E. Oberla, A. Vostrikov, M. Wetstein, An internal ald-based high voltage divider and signal circuit for mcp-based photodetectors, Nucl. Instrum. Methods A 780 (0) (2015) 107 - 113. doi:http://dx.doi.org/10.1016/j.nima.2015.01.034

[11] H. Grabas, R. Obaid, E. Oberla, H. Frisch, J.-F. Genat, R. Northrop, F. Tang, D. McGinnis, B. Adams, M. Wetstein, $\{R F\}$ strip-line anodes for psec large-area mcp-based photodetectors, Nucl. Instrum. Methods A 711 (0) (2013) 124 - 131. doi:http://dx.doi.org/10.1016/j.nima. 2013.01 .055

[12] A. Lehmann, A. Britting, W. Eyrich, F. Uhlig, Studies of mcp properties, Journal of Instrumentation 4 (11) (2009) P11024. doi:10.1088/ 1748-0221/4/11/P11024.

[13] Z. Insepov, V. Ivanov, S. Jokela, M. Wetstein, Comparison of backscattering properties of electron emission materials, Proc. of Part. Accel. Conf. TUP005 (2011) 1-3.

[14] J.-F. Genat, G. Varner, F. Tang, H. Frisch, Signal processing for picosecond resolution timing measurements, Nucl. Instrum. Methods A 607 (2) (2009) 387 - 393. doi:http://dx.doi.org/10.1016/j.nima.2009.05.193

[15] S. Ritt, The role of analog bandwidth and S/N in timing (talk), Workshop on The Factors that Limit Time Resolution in Photodetectors, U. Chicago. 
URL https://psec.uchicago.edu/workshops/fast_timing_conf_ $2011 /$

470

475

[18] B. Adams, M. Chollet, A. Elagin, E. Oberla, A. Vostrikov, M. Wetstein, R. Obaid, P. Webster, Invited article: A test-facility for large-area microchannel plate detector assemblies using a pulsed sub-picosecond laser,

480 Review of Scientific Instruments 84 (6). doi:http://dx.doi.org/10. 1063/1.4810018.

[19] C. Craven, Incom, Inc, private correspondence. 
LaTeX Source Files
Click here to download LaTeX Source Files: LAPPDTiming.zip

LaTeX Source Files
Click here to download LaTeX Source Files: LAPPDTiming.zip

Click here to download LaTeX Source Files: LAPPDTiming.zip

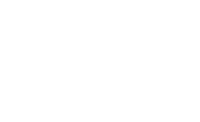
$\sqrt{10}$ (1) $\sqrt{3}$ (1)

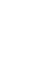
(1) (1) $\sqrt{3}$ . . .

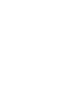

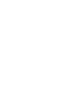

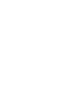

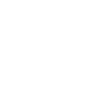

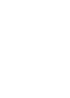

(1) .

(1) (1) . . . . 列 . 列

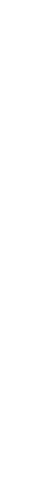
.

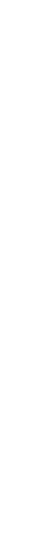

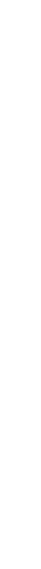
(1) (1)

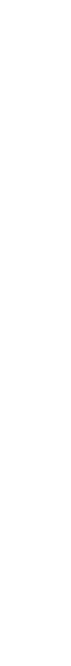
(⿸丆口

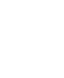
.

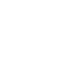

